\title{
Statistical Quality of Service to Increase Qos/Qoe of IP-Based Gateway for Integrating Heterogeneous Wireless Devices
}

\author{
Pon. Arivanantham, \\ Research Scholar, Sathyabama University, \\ Chennai
}

\author{
Dr. M. Ramakrishnan \\ Professor, Department of Information \\ Technology, Madurai Kamaraj University
}

\begin{abstract}
In broadcast service area above communications supported cellular wireless networks, data is communicated to several addressees from a right of entry point/base station. Multicast significantly progresses the network effectiveness to dispense data to multiple addressees as associated to multiple unicast gatherings of the similar data to each receiver independently, by taking improvement of the communal nature of the wireless intermediate. These algorithms need to be intended to be responsible for the essential Quality of Service (QoS) towards an extensive assortment of applications while permitting seamless roaming between multitudes of access network technologies. This paper proposed a cellular-aided mobile ad hoc network (CAMA) structural design, in which a CAMA representative in the cellular network accomplishes the control data, while the data is transported over the mobile terminals (MTs). The routing and security info is substituted among MTs and the negotiator over cellular radio channels. A location centered routing protocol, the multi-selection greedy positioning routing (MSGPR) protocol, is projected. This novel feature makes it more appropriate in the actual world. In accumulation, dynamic new call blocking possibility is initially familiarized to make handoff decision for wireless networks. This paper proposes a novel technique to afford QoS sustenance by means of an assistant network to recuperate the failure of multicast data in the major network. A wireless device might misplace some of the multicast records send above the major network. The experiment results have exposed that the proposed algorithm outclasses traditional algorithms in bandwidth deployment, handoff dropping rate and handoff rate.
\end{abstract}

Keywords-Heterogeneous Wireless Mobile Networks; Ad Hoc Networks; Cellular Networks; Quality of Service; Security; Wireless Networks

\section{INTRODUCTION}

Upcoming wireless technology aims at providing a sunshade of services to its operators. Ad hoc systems have developed attractive for their potential for commercial relevance. Routing within ad hoc network is a challenge caused by the mobility of clients and the lack of fundamental control. Several routing protocols are proposed. These approaches agonize in network presentation that consists of large routing directly above, low throughput, and large end-to-end interruption. During ad hoc networks, the concerns of quality of service (QoS) and precautions are extra convoluted for the reason that lack of reliable approaches to allocate information in the entire network. With the accumulative admiration and demand for wireless connection-based multimedia services and the erudite competences of mobile devices, mobile multimedia multicast/broadcast services have developed a significant element of wireless system. Individually the majority of significant features of wireless multicast are highly well-organized interactions as of the mutual environment of the wireless medium. At present 2.5G and 3G cellular networks are contribution multimedia services similar to Mobile TV. Broadcasting networks particularly intended for mobile communications like DVB-H [1], DMB [2] along with Media FLO [3] are presently further down deployment. In accumulation to these devoted mobile broadcasting networks, 3G cellular systems have been protracted to support Multimedia Broadcast Multicast Service (MBMS) [4]. One experiment in provided that such services be towards assurance the reception dependability of many multicast receivers since the wireless links are inaccuracy prone and numerous receivers knowledge heterogeneous channel circumstances.

The multicast service area in several networks for example Digital Video Broadcasting (DVB) and 3G multimedia broadcast/multicast facilities [5], [6] do not afford any opposite communication channel for the receivers to demand the retransmission of lost data. In several wireless multicast/broadcast systems, forward error correction codes (FEC) are cast-off to preserve multipath fading interference and decrease the package faults. Though the wireless channel circumstances are period varying and the multiple receivers in a multicast involvement heterogeneous channel environments. The FEC codes are frequently intended for the poorest channel conditions to confirm satisfactory reception quality for all the receivers in the anticipated service area. This results in a huge above in expressions of radio possessions in infrastructure-based multicast networks. Additional method to progress consistency and throughput is to use numerous projections. Conversely, this method suffers high cost and difficulty for wireless structures at the base station and the wireless devices. Consequently it is a key and stimulating task to sustenance good quality multicast provision to multiple mobile receivers while capably developing radio resources and refining the data and QoS of infrastructure-based cellular wireless networks. 


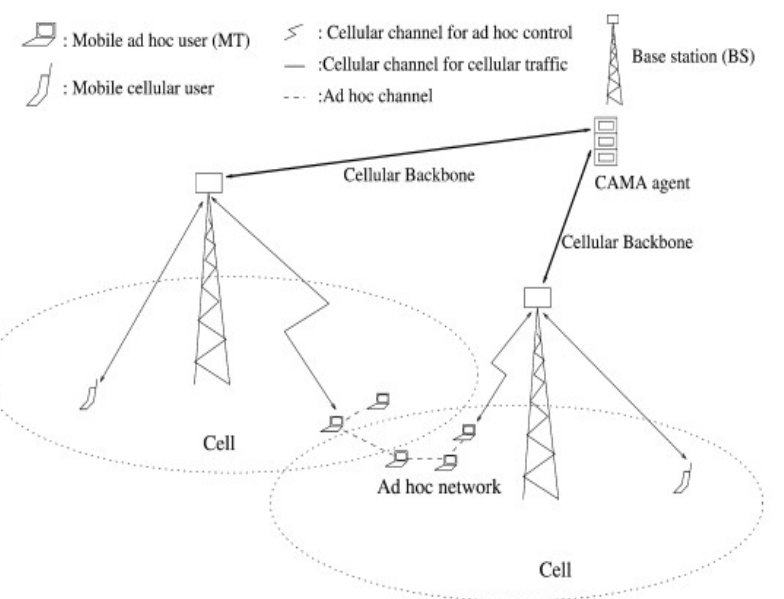

Fig. 1. Cellular-aided mobile ad hoc network

In this manuscript we are propositioning a solution through an integrated categorization uncontainable to packet loss, to provide high-quality multicast services over wireless networks.

The proposed structure contributions the major communications network (e.g., 3G) by enthusiastically conception an accommodating associate recuperation network (e.g., WiFi) between neighboring devices that make use of the identical service. For each device is fortified with two physical radio crossing point. One crossing point is associated to the major network and is answerable for receiving the downstream multicast statistics. The supplementary interface is cast-off to setup the subordinate recovery network. The devices recuperate missing multicast data packets in their foremost network by demanding them as of their recovery network. A device, by originating a supportive recovery technique in its subordinate recovery network, requirements and receive missing data from the devices that are in the locality. These adjacent devices use the identical service and have appropriately established the specific data packets. In this manner the devices recuperate lost data and therefore increase the QoS for the specific service.

\section{RELATED WORK}

Numerous methods have been proposed to develop the quality and productivity of the cellular network/ infrastructure with the help of an ad hoc network. In the reporting system [7], and mobile devices with superior quality link to work as base station transmit for devices with poor link quality. In this classification, is used as an interface to a wireless one for each of the relay and infrastructure method. Bounded by the total cell productivity achieved in this hybrid network of display cellular bandwidth available. Recent reports in the system [8], and used two types of interfaces for wireless ad cellular networks, especially for the integration. In this scheme, and use wireless high bandwidth channels in ad hoc mode (IEEE 802.11) for the transfer of a single-traffic transmissions from the cellular network (3G) cellular to improve productivity and the extent of coverage.

System in the third reported [9] [10], and the transfer of multicast data to a node relay-wide and short inside the cellular network (3G) and is routed to the remainder of the decade Subscribe relay node via a dedicated high-speed networks (IEEE 802.11). In [11] System publishes dedicated relay devices to communicate traffic from one cell to an additional movement, to evade the difficulty of congestion due to unstable movement in the cellular system.

Been integrated heterogeneous wireless networks are applied widely and studied. Example of the application of integrated methodology is AMPS / IS-95 cellular network, and Global Positioning System (GPS) apply in the cellular network to afford services point, and satellite / cellular network [15.42]. There is also a rising significance in cellular and wireless LAN Network Integration (WLAN). Universal Mobile Telecommunications System (UMTS) [12,20,41], otherwise known as cellular network $3 \mathrm{G}$, is capable to afford dissimilar services (voice and data services) on their individual. Conversely, because of the bandwidth limited radio, and the network cannot contain a huge number of users at on one occasion, particularly for application that necessitate fast data transport rate. Additionally the high cost of the examine. As a complement to the cellular network, WLAN might afford services with high data transport rate of a moderately low cost. Combination of these two heterogeneous network be able to make available superior service by having mobile user handoff back and forth among the networks to attain the essential services [33.34] and [17]. Conversely, WLAN has a extremely small radio coverage (particularly in urban areas), and can simply afford services to users is very secure to its fixed access point. To be capable to serve most users in such an included network, this will be posted on the high density of access points for wireless networks. This leads to increased cost and reduced fixed infrastructure efficiency. To overcome this obstacle, a dedicated network can be used instead of the WLAN. In / integrated cellular network dedicated and multi-hop ad-private links stretching almost radio coverage.

It also can access the mobile phone client outer surface the radio coverage of the service access position (fixed or mobile) throughout an intermediary routing. Cannot peer-to-peer service is accomplish directly throughout a dedicated network without going throughout the cellular network. In addition, you can use a custom to redirect traffic between the cells to get the budget in the cellular network channels. This further improves the cellular network's capacity. Research/ cellular network integrated custom can be found in [17.45], and [46]. This effort spotlight on how a enthusiastic network may improve cellular services. Perhaps called the ad hoc advance with the assist of cellular networks. Global Positioning System (GPS) [1,35] has been used extensively to serve GPS. Based on top of satellite signals established, you may point to conclude its position throughout a built-in GPS chip. Among the help of GPS, which is the foundation of MT might recognize where the objective is the MT and decision making suitable assistance. And called such a point with the assist of GPS assistance for positioning technique. Studying greedy perimeter stateless routing 
(GPSR) in [12]. In GPSR, the subsequently stage of the path is constantly closest to the intention MT. And MT requirements to be acquainted with the exact position of the multilateral trading system and all other parties. Authors make the assumption that there is a server position. In [22], presumably on the MT source to find out the destination MT position. Not flooded routing requests in the network, but only directed towards the destination to reduce the routing overhead. In [19], and GPS guidance similar to those in [22] are the study protocol. Other works on the help of GPS guidance can be found in [26.28]. Management is considering the situation in detail in [7, 25]. In [25], distributed server model site is described. MT multilateral trading system and uses the other parties within a certain area as its servers. MT position will be sent to these site servers periodically. Can multilateral trading system and the other parties know this position MT through access to any of its servers. In another approach to manage the site [7], all MT has a virtual home region (VHR) with a fixed center. MT and update their locations by sending ads stand for VHR her. In all the newspapers, the multilateral trading system must know the approximate coverage customized for the network. There are over a relatively large presence of site updates. In an environment where GPS is not available, such as an internal office, information can be used to determine the relative positions by custom to select the routing network. It utilize an algorithm to conclude the self-sites to compute the comparative positions for MTS in [7], so that the synchronize system network can be built on the site in sequence. In an additional paper [43], the authors recommend routing performance founded on the link among proposed for users more willingly than particular sites.

This routing protocol is known as association beaconing routing (ABR) protocol. In the majority obtainable ad allocated routing protocols, there is a message "hello", which might assist to attain information as regards the MT neighbors. Though, it is complicated to association condition routing in ad hoc networks due to the dynamic topology and distribution of information slow. In previous work, there is no central server specific provision of global information, so it can not apply the directive sites based on the information the global position of minutes.

\section{PROPOSED WORK}

In this research, we concentrate the significant problem of performing routing by means of end-to-end statistical QoS assurance in heterogeneous MANETs. As of previous discussion, we examine that QoS routing in MANETs engage two key in sub problems: (1) Generate a stable and connected routing structural propose that is robust in opposition to MANETs frequent topology modify and link failure, and (2) performing arts QoS routing on the consequential virtual spine. We concentrate on each of these two key sub problems individually, while at the equivalent time provided that an integrated clarification for the QoS routing difficulty in MANETs.

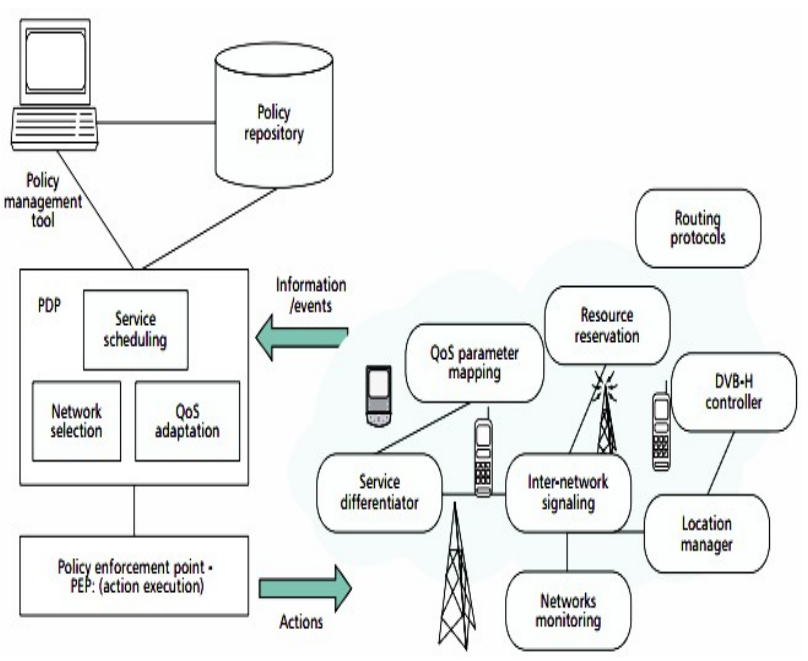

Fig. 2. System architecture of the Policy-based USG

USG is a compilation of the subsequent four types of entities:

- Policies: policies can be disseminated crosswise the UWD nodes depending on their implementation scopes;

- A policy assessment engine that motive over these strategy (known as the strategy assessment point or PDP in IETF expressions[5] to make decisions on network assortment and QoS adaptation;

- An accomplishment implementation engine (known as the strategy enforcement spot or PEP in IETF expressions) that afford the genuine execution of policy actions;

- Sequences of apparatus that execute the network procedure or pattern such as service discrimination, QoS parameter mapping, inter-network signaling, routing, and so on.

These apparatus are position across the UWD network. The adaptableness of this system structural design comes from its consciousness of the process surroundings (or context information).To makes an inclusive decision, the USG must reflect on the subsequent factors: service types, user necessities, user device individuality, and network status. These factors are all articulated by policies. Re-routing (including route repairing) or network reconfiguration transpire when there is a important modify in definite factors. This adjust is reported to the PDP throughout an occasion, and the PDP makes a appropriate decision by calculation above the policies. The way of thinking procedure might consist of variance resolution if the new background changes result in inappropriateness with user or system necessities. The events also can be generating by a instance phase circumstance or as a result of an action. Reinforcement the PEP is a variety of network administration and routing function. Those explicit to UWD service provisioning are in briefly converse as follows. For other common apparatus, pass on to the debate in [7]. 
Our involvement in this research paper is two-fold. Primary, we recognize that the majority of the routing problems in MANETs are partially due to the unbalanced topology of these networks. Consequently, having a predetermined, stable, and associated routing structural design (i.e. virtual wireless backbone) that is robust against node progress and recurrent link breakdown in MANETs can make simpler those routing tribulations. We examine provide such a constant topology with low transparency. For this principle, we develop an original zoning stratagem for heterogeneous MANETs. The zoning method maps the network objective topology on top of a virtual two-dimensional grid topology. The virtual grid consists of the occasional, but perhaps more commanding, mobile nodes called as Cluster Heads (CHs), which are designated sporadically. The proposed fasten effective topology provides stable routing backbone that is castoff to execute routing with the requisite end-to-end QoS assurance. Second, we proposition a QoS routing protocol for heterogeneous MANETs. The proposed protocol, called virtual grid architecture protocol (VGAP) makes use of cross-layer devise methodology with the intention of end-to-end QoS assurance. VGAP functions on the derivative predetermined virtual grid, where CHs ascertain various QoS routes by means of an comprehensive adaptation of the OSPF routing protocol, called Mobile OSPF (M-OSPF). To calculate QoS routes, M-OSPF utilize an comprehensive version of WFQ development policy, called Ad hoc WFQ (AWFQ). AWFQ takes into account the anecdotal time uniqueness of the wireless direct state into version, yet providing an higher vault on the arithmetical end-to-end delay guarantees of a definite flow. The prospect of using OSPF as a routing protocol in MANETs has been in current times discussed by the IETF MANET collection [24] but with no particular particulars.

\section{NETWORK MODEL AND VIRTUAL NETWORK TOPOLOGY}

We mull over a heterogeneous network that consists of $\mathrm{N}$ mobile nodes of dissimilar potential that are arbitrarily dispersed in the network area. To exploit location in sequence, we presume that each mobile node is operational with a global positioning system (GPS) card.

\section{A. The zoning process}

For the sake of effortlessness, but without loss of generalization, we presume that mobile nodes are of two dissimilar broadcast ranges: $r_{\mathrm{s}}$ for a short range (SR) nodes and $r_{l}$ for long range (LR) nodes. The broadcast assortment in each case is a utility of the energy level at the mobile node. The network area is separated into fixed, disjoint, and standard shape zones (clusters). To generate a easy rectilinear virtual topology, we decide on the sectors to be square in form. A sector might have a combination of SR and LR nodes. At first, the network region is separated into large sectors where the sector side length $\mathrm{x}_{1}$ is selected such that two LR mobile nodes in adjacent horizontal/vertical sectors and placed anywhere in the sector can correspond with each other straightforwardly. Consequently, $x_{1}$ is preferred as $x_{1}=\eta / \sqrt{5}$. If the sector has only short range nodes, the sector is separated into subzones where each subsector side length $\left(\mathrm{x}_{\mathrm{s}}\right)$ is calculated as $\mathrm{x}_{\mathrm{s}}=\mathrm{x}_{1}=2$ :
The numeral of layers in the fundamental topology is resolute by the number of broadcast series in the network. In Fig. 3 (b), the left side sectors have equally SR and LR nodes while the right side sectors have only SR nodes. Consequently, each of the right side zones is separated into four subzones to allocate straight communication among any two short range mobile nodes in adjoining horizontal/vertical subzones. As a consequence, the control visual projection is inadequate to the communication among $\mathrm{CHs}$ and in the linked vertical and horizontal directions only.

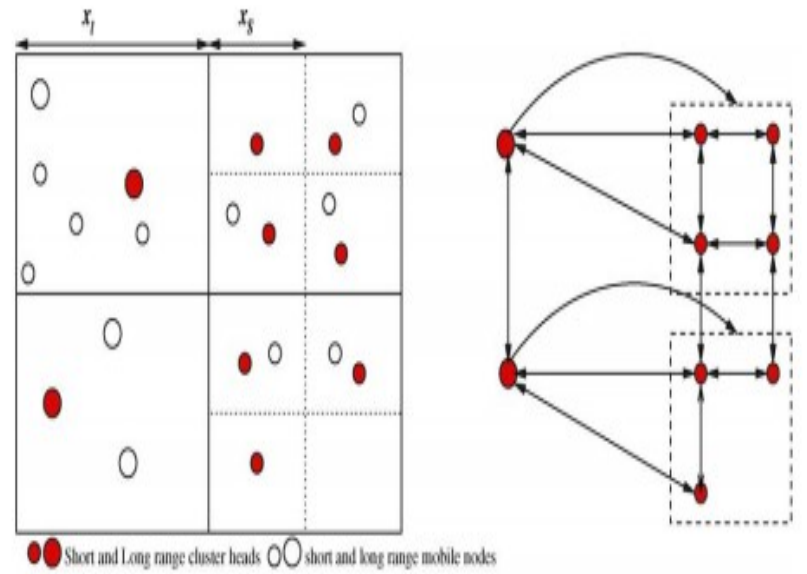

(a) Zoning with physical topology (b) Corresponding virtual topology

Fig. 3. Network zoning and the virtual topology

\section{B. Cluster head election}

Since mobile nodes be different in their potential, an eligibility criterion is considered necessary to establish which mobile node can develop into a $\mathrm{CH}$. reminder that parameters like remaining battery power, node speed, and node position be supposed to count up in the direction of this decision. The $\mathrm{CH}$ role is revolved occasionally in the middle of nodes in each zone. The $\mathrm{CH}$ periodicity assists to equilibrium the nodes load allocation, attain fairness, and afford fault tolerance in opposition to single node failure. We describe the time flanked by two consecutive $\mathrm{CH}$ election instantaneous as the $\mathrm{CH}$ election interlude. each one mobile node make a decision whether it will become a $\mathrm{CH}$ for the present election period based on an eligibility factor (EF). The least mobile, most power proficient, most location suitable, and most lightly loaded node is the best applicant to act as $\mathrm{CH}$. The eligibility of a node $\mathrm{i}$ to serve up as a $\mathrm{CH}$ at time $\mathrm{t}$ can be considered as follows:

$$
E F_{i}(t)=a_{1} e^{-v_{i}(t)}+a_{2}\left(1-Q_{i}(t)\right)+a_{3} B_{i}(t)+a_{4}\left(1-s_{i}(t)\right)
$$

Wherever

$-v_{i}(t)$ is the standard speed of node $\mathrm{i}$ at time $\mathrm{t}$.

$B_{i}(t)$ is the outstanding power in node i battery at time $t$,

$Q_{i}(t)$ is the tiny proportion of time node i remainder as $\mathrm{CH}$ for the duration of a time windowpane $\mathrm{T}$ (defined as a windowpane of time adequate to capture cluster dynamics) end at time $\mathrm{t}, 0 \leq Q_{i}(t) \leq 1$, and establish as follows 
$Q_{i}(t)=\frac{1}{w} \sum_{m=1}^{w} Y_{i}(t-m \delta t)$

where $\mathrm{Yi}(\mathrm{t})$ is a binary indicator which is 1 if and only if Wherever $\mathrm{Yi}(\mathrm{t})$ is a binary pointer which is 1 if and only if node $\mathrm{i}$ was a $\mathrm{CH}$ at time $\mathrm{t}$, and $\delta \mathrm{t}$ is a diminutive time percentage enhance such that $\mathrm{T}=\mathrm{W} \delta \mathrm{t} . \mathrm{S}_{\mathrm{i}}(\mathrm{t})$ is the Euclidean detachment of node i regarding the midpoint of a zone at time $t$, and $a_{1}, a_{2}, a_{3}, a_{4}$ are scaling feature that replicate the significance of each parameter. Mobile nodes in each one zone will switch over the values of the intended EF's. The node that has the uppermost value of $\mathrm{EF}$ will decide on itself as the $\mathrm{CH}$ for the present election phase. That is, if a zone $\mathrm{z}$ has a nonempty set of $\mathrm{n}$ nodes, the $\mathrm{CH}$ is elected as pursue.

$$
C H=\arg \max _{i \in n}\left(E F_{i}\right)
$$

Each mobile node that has selected itself as a $\mathrm{CH}$ for the present phase will transmit a commercial message to situate the mobile nodes in its zone. If the $\mathrm{CH}$ foliage its zone or fails early, an early determination of the next $\mathrm{CH}$ obtain position after a handoff process in which the new $\mathrm{CH}$ succeed to the routing accumulation of the retreat $\mathrm{CH}$. It is value declaring that the conception of $\mathrm{CH}$ determination is not a new conception. Conversely, our zoning method reduces to bare bones of its operation. Consequently, the routing utility will also be simplified.

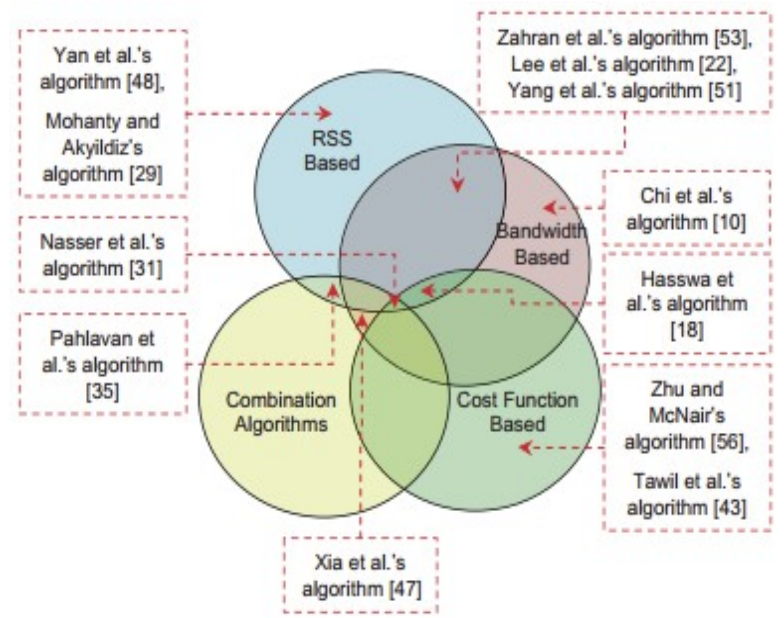

Fig. 4. The four categories of VHD algorithms and twelve selected representative schemes

\section{Mathematical derivation of TBVH}

In this section we begin by exploring the different scenarios for the time before a vertical handover (TBVH) component for upward vertical handovers, based on the MH's location and direction of movement. For the sake of simplicity, in this paper we only consider the UMTS-WLAN handover combination, although our model is easily applicable to other network combinations as well.

The first scenario to be considered is a $\mathrm{MH}$ that is roaming under the coverage of a BBS and is moving towards the boundary with velocity $v$ as shown in Fig. 1 . Here we consider a circular cell of radius $\mathrm{R}$. The inner dotted concentric circle shown in the above figure represents the handover threshold of radius $r$ which is the distance from the BSS where a $\mathrm{MH}$ is expected to perform a handover. Location co-ordinates of the MH can be extracted as explained in references [3,11]. While $\mathrm{x}$ is the angle made by the BBS and direction of movement at the $\mathrm{MH}$, the distance $\mathrm{d}$ between the BBS and $\mathrm{MH}$ and $\mathrm{r}$ can both be derived from the equation where the Received Signal Strength (RSS) is given by [14]:

$$
R S S_{d B}=-10 \gamma \log (l)
$$

Where $l$ is distance from the transmitter and $\gamma$ is the propagation path-loss coefficient. The BBS passes the latest values of the required information to the $\mathrm{MH}$ which is responsible for performing the calculations. In order to find the TBVH in this scenario we need to calculate the distance $Z$ which is the point on the threshold circle where the $\mathrm{MH}$ is expected to vertically handover. As

$$
r^{2}=d^{2}+z^{2}-2 d z \cos x
$$

The value of $Z$ calculated by solving the quadratic equation is

$$
z=d \cos x \pm \sqrt{r^{2}-d^{2} \sin ^{2} x}
$$

Therefore, the estimated TBVH for this scenario is:

$$
T B V H=\frac{d \cos x+\sqrt{r^{2}-d^{2} \sin ^{2} x}}{v}
$$

The parameter values in the above equation can be easily obtained, making it possible to calculate TBVH.

As WLANs may have specific points of exits such as doors in a building, the prediction accuracy of TBVH can be improved if the co-ordinates of these exit points are stored in the BBS and passed on the MH which then calculates the TBVH to the exit point.

In this case we adopt the concept of threshold distance TD [9] in the normal BS. This is a distance smaller than the cell's radius, which describes a smaller concentric circle located within the cell. The idea is that a MH moving inside the TD circle is more likely to change its direction, however on moving out of this circle, it is less likely to undergo a sudden change in its direction, thus enabling a correct prediction of the cell the MH is moving towards.

The goal in this case is to improve the prediction capability of the model by making it able to predict the TBVH of $\mathrm{MH}$ while it still moves in the coverage of $\mathrm{A}$. As the $\mathrm{MH}$ is too far from the BBS to get a reasonably accurate value of $b$, we first need to find this distance and the angle $\beta$ in order to calculate distance $Z$.

$$
c^{2}=d^{2}+b^{2}-2 d b \cos \theta
$$

Therefore, 


$$
\theta=\cos ^{-1}\left(\left(c^{2}-d^{2}-b^{2}\right) / 2 d b\right)
$$

Depending on which side of line $\mathrm{AB}$ point $\mathrm{X}$ lies,

Angle

$$
\beta=|x-\theta|
$$

Considering triangle BYC, we have

$$
\begin{aligned}
& t=b \cos \beta \\
& y=b \sin \beta
\end{aligned}
$$

Therefore, in triangle BYX,

$$
s=\sqrt{r^{2}-b^{2} \sin ^{2} \beta}
$$

As $Z=t+s$,

From (7), (8) and (9) we have

$$
z=b \cos \beta+\sqrt{r^{2}-b^{2} \sin ^{2} \beta}
$$

Thus the TBVH component for this scenario is,

$$
T B V H=\frac{b \cos \beta+\sqrt{r^{2}-b^{2} \sin ^{2} \beta}}{v}
$$

This is similar to the equation obtained in (4).

\section{Positioning Routing IN CAMA}

\section{A. Centralized positioning routing}

In CAMA, positioning routing is more reasonable because the CAMA representative may work as a centralized positioning in sequence attendant. An MT can locate its precise geographical point throughout GPS 1 . The point in sequence is send to the CAMA representative throughout the BS. An MT's point can as well as establish by the cellular network by means of the current cellular position service. Separate from the positioning routing used in the wholesome ad hoc network, in CAMA routing, the present point of each MT can be fine known. An preliminary route from a resource to a objective can thus be indomitable moreover by CAMA agents or by MTs. If the routing is resolute by MTs, the BS will have to transmit the most efficient point in sequence. Founded on the conventional in sequence, each MT makes its hold routing verdict.

In this paper, CAMA agent is measured to be assembly the routing decision. Compared to MTs, the CAMA agent has further absolute global in sequence for the complete ad hoc network. This centralized routing method also transport advantages of routing optimizations, precautions, radio resource distribution and power savings. Furthermore, the centralized routing system doesn't require the episodic downlink dissemination of the positioning in sequence which generally devour large cellular radio bandwidth and MT power. Conversely, the centralized control has its drawback. An MT might have to remain for a extensive time to obtain the routing decision from the CAMA agent if a lot of MTs send routing requirements at the similar instance. The delay is essentially caused by the retreat delay due to the uplink transmission conflict. In the case of a CAMA agent portion MTs in excess of one cell, the delay also comprise the uplink demand queuing delay and the downlink respond queuing delay. If the downlink delay is too elevated, the direction might lose its contemporariness. A new-fangled method has to be resolute by the simplified position in sequence. As of the standpoint of precautions, the centralized routing decision method might endure the attack of denial of service (DoS). In a actual wireless network, particularly in the inner-city area, two geographically secure MTs may not attain each other by the use of radio because of the composite radio proliferation environment (e.g., radio block). To develop the GPS routing exactness, MTs can send "hello" messages to their neighbors to confirm they are accessible to each other. This organization statistic is sent to the CAMA agent among MTs' particular positions, in order that the CAMA agent might know precisely what link exists, and make more exact routing assessments. This, conversely, enhance the overhead in mutually the cellular network as well as the ad hoc network. It must be illustrious that based on correlation between MTs, the CAMA agent might also make the routing decisions throughout link state routing techniques. The technique is not as instantly onward as GPS positioning routing and will be deliberate in our future work.

\section{B. The procedure for making routing decisions}

While an MT needs to send information to its objective, it will send a routing demand to the CAMA agent throughout the cellular radio channel. The channel know how to be the unsystematic access channel, the uplink regular packet channel, or else a pre-assigned traffic channel in UMTS. CSMA/CD how to be the unsystematic access method for the cellular uplink access. The MT will re-send the routing demand if it doesn't take delivery of the routing respond later than a time-out. The failure to receive a routing pronouncement is basis essentially by conflict with the concealed MTs. Conversely, the hiding workstation trouble here is not as severe as to facilitate in WLAN ever since the cellular radio reporting is huge enough measure up to the size of ad hoc network. The CAMA agent responds to the MT by means of a absolute route as well as every transitional MT throughout the frontward access channel, the downlink mutual channel, or else a pre-assigned traffic channel of the cellular network. Given that the point of all the MTs are recognized, the detachment for each hop is too recognized and the communication power of every MT can be predictable.

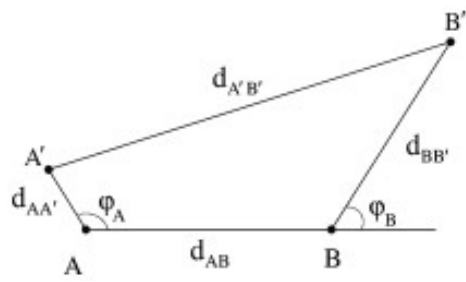

Fig. 5. An example of movements for ad hoc MTs 
To supplementary accumulate power, MTs might “sleep” although listen to the cellular channel (e.g., the transmit channel or else the paging channel) every so often when they are not integrated in any active routes. When a original routing assessment is completed, the BS will page all the transitional MTs on the route by means of the objective MT by dissemination their IDs. These MTs will "awaken" to obtain and broadcast the data packets. In spite of everything the packages are received by the objective, the route will be free with all MTs on the route "sleep" over again. The routing in sequence is conceded in the description of each one data package, as is in DSR [8]. The midway MTs read the routing assessment to locate their next hop, as well as the suggested transmitting supremacy.

\section{Position update}

Location update is required when an MT progress on or after its earlier location. Intended for the GPS-aided positioning direction-finding, an MT has to launch its new-fangled position to the CAMA agent throughout the cellular channel. The new-fangled positions are modernized occasionally, by means of a time threshold value for the update phase. This value is founded on the specified prospect of wrong routing choice attributable to out-of-date position data being an end to than a value p $\tau$ t. It essentially depends on the network traffic, i.e., how frequently a original route has to be resolute and how frequently an MT is integrated in a new route.

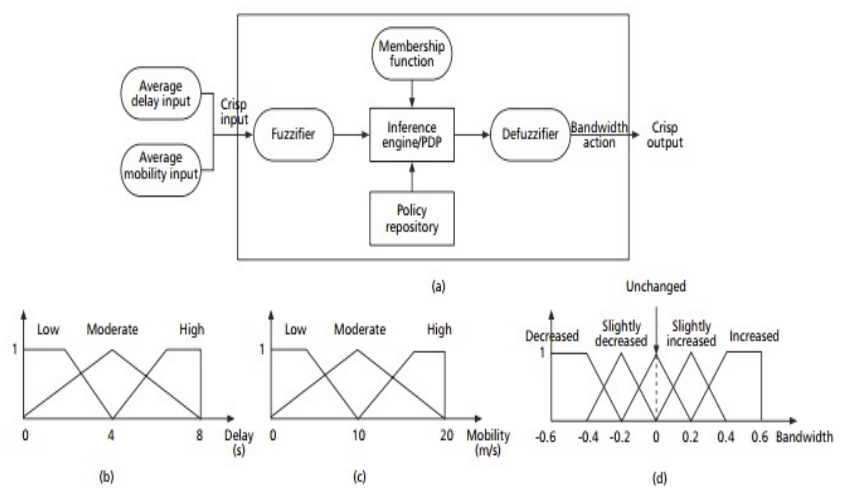

Fig. 6. The architecture of: a) the Fuzzy Control Module; and b-d) membership functions

Intended for the policy-based USG to endure the formless and indistinct data, fuzzy logic and fuzzy control [10] is make use of in group effort with policies and the PDP. The beginning of fuzzy control facilitate policies in the USG structure to utilize linguistic verbal communication to illustrate a network pattern tutoring, for model, "IF average delay is restrained AND standard mobility is low down, THEN bandwidth have to be improved." Nevertheless, the delay assessment reported through the network is a hard value such as $500 \mathrm{~ms}$, and the network can simply recognize hard values for bandwidth for example $1.5 \mathrm{Mb} / \mathrm{s}$. For itself, there is a constraint for fuzzification and de-fuzzification as exemplify in the USG fuzzy control unit exposed in Fig. 5a. Intended for the present grade of our research, we choose three delegate network limitations to utilize for fuzzy control: delay, mobility, and bandwidth. The equivalent principle and process are appropriate to further network parameters. The linguistic variables used to characterize the standard transmission delay of a data package are: low, moderate, and high. Those to characterize the standard node mobility as well as: low, moderate, and high. In the strategy point out earlier, both delay and mobility materialize in the provision part of the strategy. The fuzzy variables used to signify the strategy action, that is, bandwidth distribution, are separated into five levels: unchanged, a little increased, increased, a little decreased, and decreased. Intended for network pattern associated to delay, mobility, and bandwidth, the subsequent $3 * 3=9$ policies or system, in the middle of several others, are necessary through the fuzzy control unit. Ever since triangular and trapezoidal formed membership task recommend more computational effortlessness than a Gaussian function [10], we select these to classify the membership task for the preceding three fuzzy variables worn in the policies. The realistic intend of these membership functions is fundamentally application definite. During our bandwidth allotment circumstances, we distinct the membership functions for delay, mobility, and bandwidth respectively as shown in Fig. 5b-d. The degree of membership is determined by plugging the monitored input parameter (e.g., delay or mobility) into the horizontal axis and projecting vertically to the upper boundary of the membership function(s). For example, if the input for delay is one second and the input for mobility is $9 \mathrm{~m} / \mathrm{s}$, then the membership functions show that the input linguistic value is low and moderate, respectively. The output value from the fuzzy control module activates the adaptation choice that is slightly decreased according to the corresponding linguistic policy. Then this fuzzy output response must be de-fuzzified by using the output membership function for bandwidth given in Fig. 5d. As a result, a crisp value for a decrease in bandwidth can be deducted, for example, decreased by 20 percent. An inference process is involved, especially for cases with more than one policy whose actions are satisfied. The MAX-MIN method [10], which tests the magnitude of each policy and selects the highest one, is adopted in our implementation. This method, though not combining the effects of all applicable policies, does produce a continuous output function and is easy to implement.

It is possible that when it is time for an MT to update its position, it remains close to the position in its previous update. A new position update is not necessary since there is no change in routing topology, and position update brings signaling and operating load to the cellular network. To determine whether a position update needs to be sent, another threshold value of the distance between an MT's updated position and its position during last update should be defined. This threshold value is based on the requirement that the probability of a one-hop link break due to the non-updated position information should be no greater than $\mathrm{p} \tau \mathrm{d}$. It should be adaptive in the networks with different MT mobility patterns. The threshold values can be estimated mathematically. To determine the time threshold value, we assume that $\lambda$ is the mean of a Poisson packet arrival to each MT, and $m$ is the average number of hops in each link. The time interval between any two cases in which an MT has to be active in a route is approximately negative exponentially distributed with a mean of $1 /(\lambda \times \mathrm{m})$, so that the time threshold 
value can be calculated by solving the equation $1-e^{-\lambda m t \tau}=p_{\tau t}$. For the distance threshold value, we assume that the original positions for two connected MTs are A and B. After a while these two MTs move to the new positions which are A' and B' respectively, as shown in figure 4 . The new distance between these two MTs, dA'B', is:

$$
d_{A^{\prime} B^{\prime}}=\left(\left(d_{B B^{\prime}} \cos \varphi_{A}+d_{A B}\right)^{2}+\left(d_{B B^{\prime}} \sin \varphi_{B}-d_{A A^{\prime}} \sin \varphi_{A}\right)^{2}\right)^{1 / 2}
$$

Assume $d_{A B}, d_{A A^{\prime}}, d_{B B^{\prime}}, \varphi_{A}, \varphi_{B}$ are independent random variables with known distributions, the probability density function (PDF) for $d_{A^{\prime} B^{\prime}} \leq r$ under different distance threshold values can be numerically calculated, where $r$ is the maximum ad hoc radio coverage. From the PDF function, we can find the threshold value $d_{\tau}$ for $d_{A A^{\prime} \text { and }} d_{B B^{\prime}}$ so that $p \leq p_{\tau d}$. A numerical result of the percentage of a link-break against different distance threshold values is shown in figure 5 .

Special updates may be needed when the radio environment for an MT changes significantly (e.g., when an MT turns a corner or goes into a building). These changes can only be measured by sending "hello" messages between MTs for reachable neighbors.

\section{METHODOLOGY}

\section{A. The First Step: Quick Evaluation Method for the Pre-Handoff Decision}

Without a doubt, unusual classes of services necessitate different mixture of vertical handoff limitations (e.g., reliability, latency, and data rate). Consequently, user's traffic module (i.e., classes of service, service types) must be well thought-out in the handoff decision. Intended for the traffic classes, we track the four QoS classes of network applications distinct by UMTS [21]. They are informal class, streaming, interactive class and background class. For instance, according to the delay compassion distinctiveness, the primary two types are clustered as instantaneous service, Whereas the further two belong to non-real instant service. Given that real-time service is susceptible to delay, a assured broadcast rate is necessary. Certainly, the separation of traffic classes can be ended by users.

In vertical handoffs, several network limitations encompass an effect on whether otherwise not a handoff be supposed to take place. The significant parameters consist of quality of service (e.g., handoff delay, accessible bandwidth), security, power supplies, cost of service, and so forth.

During the primary step, the pre-handoff verdict estimates whether the bare minimum guarantee of a abuser is sustained for each network i, i $=1,2, \ldots, \mathrm{N}$. Further exclusively, the values of a little simple detected and critical parameters ought to be additional than the predefined thresholds, in that order.

$$
\begin{aligned}
& M_{i}=F\left(b_{i}-b_{t h}\right) \cdot F\left(R S S_{i}-R S S_{t h}\right) \cdot F\left(V_{i}-V_{t h}\right) \\
& \times F\left(t_{i}-T_{t h}\right) \cdot F\left(P_{i}-P_{t h}\right) \cdot F\left(C_{i}-C_{t h}\right)
\end{aligned}
$$

Equation (6) correspond to a minimum guarantee function, which point out the minimum guarantee of MN is maintain intended for each network i. It is aimed at assembly utilize few of the limitations referred in Eq. (6) with the intention of make a earlier and wiser handoff decision. At this point $b_{i}, R S S_{i}, V_{i}, T_{i}, P_{i}$ and $C_{i}$ characterize the standards of obtainable bandwidth, received signal strength (RSS), velocity, duration, battery power and monetary cost of $\mathrm{MN}$ as of a exacting network $\mathrm{i}$. The interval Ti represent the predictable point MN will continue in a specific network $\mathrm{i}$, which is calculated by some comprehensive parameters. Into the bargain, $b_{t h}, R S S_{t h}, V_{t h}, T_{t h}, P_{t h}$ and $C_{t h}$ be the predefined thresholds of obtainable bandwidth, acknowledged signal strength, velocity, duration, battery power and pecuniary cost to maintain the appealed traffic class, The demanded traffic class repeatedly is the major traffic class or the majority of important traffic category for MN, which takes up for the most part the whole time. The utility F(.) is a unit step function. The unit step function is a irregular function whose significance is nothing for negative dispute and one for positive dispute. For this reason, previously there is not less than one parameter value of MN since a specific network $i$ is lower than its threshold, the minimum guarantee function have nil value. In this casing, network $i$ is not measured as a objective network to any further extent. If the minimum guarantee function value is individual for a specific network $\mathrm{i}$, this network will be additional to the applicant network set S. Make a note of that the set $S$ is set to be blank at the establishment of every handoff decision. Ever since Eq. (6) is effortless and the limitations as of this equation can be predictable rapidly, time utilization of the pre-handoff decision is extremely low. Certainly, various parameters can promote be misplaced according to the circumstance of exact relevance (e.g., the source of $\mathrm{MN}$, the accessibility of a few parameters referred above). For instance, in a few circumstances, Ever since the interval of MN can't be predictable for a few reasons, this parameter has to be misplaced. Clearly, by establishing the interval into the minimum guarantee function, the severe ping pong effect can be proficiently removed. Thus, our advance condenses gratuitous handoffs while ever-increasing network throughput, lessening handoff delay, and keep away from connection dropping.

In addition, the velocity should be considered in the first step. For example, if MN's speed is over $100 \mathrm{~km} / \mathrm{h}$, WLAN cannot support its speed. Besides, battery power may be a crucial parameter for certain users. For example, when the battery level is low, the user may choose to switch to a network with lower power requirements (i.e., the threshold of battery power), such as an ad hoc Bluetooth network. After the pre-handoff decision is finished, according to the size of the candidate network set $\mathrm{S}$, the propose decision algorithm generally falls into three cases. One is that the set $\mathrm{S}$ is empty, $\mathrm{MN}$ remains connected to the current network. Another is that there is only one member in set S. If the sole network is the current network, MN stays in the current network; otherwise, MN decides to perform vertical handoff procedure to be associated with the network. The other is that more than one network have been added into the set S. More specifically, there is more than one network, whose minimum guarantee 
function value is one. If $\mathrm{MN}$ is a resource poor node and the current network is in the set $\mathrm{S}$, MN will remain connected to the current network. If $\mathrm{MN}$ is a resource poor node and the current network is not in the set S, MN will handoff to any network included in the set $\mathrm{S}$. If $\mathrm{MN}$ is a resource rich node, $\mathrm{MN}$ will proceed to the second step.

\section{B. The Second Step: Vertical Handoff Decision Function}

In this secondary step, an extended vertical handoff decision function (EVHDF) is existing, which is an extensive description of VHDF in [1]. EVHDF is worn to evaluate the development expanded by handing off to a exacting network $\mathrm{j}$ incorporated in the contender network set S. At this point we presume the dimension of the candidate network set $\mathrm{S}$ is $\mathrm{m}$. It is noticeable that $\mathrm{m}$ is an numeral greater than one. According to Eq. (5), it can be seen with the intention of the calculation of DNCBP doesn't simply narrate to accessible bandwidth, but also network traffic load. Additionally, the DNCBP can be worn to point out network traffic load. Consequently, utilize the DNCBP in the EVHDF can be supplementary practical for network load balancing crosswise diverse networks than supplementary parameters (e.g., obtainable bandwidth). The network by means of the uppermost intended value for EVHDF is the majority optimal for MN based on particular predilection. The EVHDF for a meticulous network $\mathrm{j}, E Q_{j}$ is defined by:

$$
\begin{aligned}
& E Q j=\frac{\omega\left(1 / c_{j}\right)}{\max \left(\left(1 / c_{1}\right), \ldots . .,\left(1 / \mathrm{C}_{\mathrm{m}}\right)\right)}+\frac{\omega_{\mathrm{s}} S_{j}}{\max \left(S_{1}, \ldots \ldots, S_{m}\right)}+\frac{\omega_{\mathrm{p}} P_{j}}{\max \left(P_{1}, \ldots \ldots, P_{m}\right)} \\
& +\frac{\omega_{D} D_{j}}{\max \left(D_{1}, \ldots \ldots, D_{m}\right)}+\frac{\omega_{\mathrm{F}} F_{j}}{\max \left(F_{1}, \ldots \ldots, F_{m}\right)}
\end{aligned}
$$

At the same time as illustrate in [1], at this point $\omega \mathrm{C}, \omega \mathrm{S}$, $\omega \mathrm{P}, \omega \mathrm{D}$ and $\omega \mathrm{F}$ are weights for every of the network parameters. The principles of these weights are tiny proportion (i.e., they range from 0 to 1 ). Furthermore, every part of five weights add together to 1 . every weight is comparative to the consequence of a limitation to the vertical handoff algorithm. In adding together, $\mathrm{C}, \mathrm{S}, \mathrm{P}, \mathrm{D}, \mathrm{F}$ there the cost of service, security, power expenditure, network circumstances, network recital, respectively. The major differentiation among EVHDF at this point and that in [1] is that $D_{j}=b_{j}$ in [1] has been substituted with

$D_{j}=\frac{b_{j}}{H_{j}}$

The network by means of the uppermost $E Q_{j}$ is the elected network. If the elected network is not the present network, vertical handoff takes places; or else, $\mathrm{MN}$ remainder associated to the present network.

\section{SimUlation RESUlTS}

We simulate an ad hoc network by means of 100 MTs reside in an region of $1000 \mathrm{~m} \times 1000 \mathrm{~m}$. Every MT move about surrounded by the area, with a unsystematic direction and a unsystematic velocity consistently distributed between 0 and a greatest value. Without any measurement, this greatest value is $3 \mathrm{~m} / \mathrm{s}$, the momentum for ordinary users. The ad hoc direct has a predetermined data rate of $1 \mathrm{Mb} / \mathrm{s}$.

The wireless crossing point workings like the $914 \mathrm{MHz}$ semi-transparent Wave LAN, with a ostensible radio range of $250 \mathrm{~m}$. MSGPR (multi-selection greedy positioning routing) underneath CAMA background is compared with two other ad hoc routing protocols, AODV and DSR. The penetrating set for MSGPR is set great adequate so that the preeminent route can always be establish. We presume that spot updates and routing requirements can constantly be sent effectively to the CAMA agent at their first effort. In this paper, the case that MTs send their links by means of adjoining MTs is not integrated.

TABLE I. SUMMARY FEATURES OF NP, QOS AND QOE

\begin{tabular}{|l|l|l|}
\hline $\begin{array}{l}\text { Quality of } \\
\text { Experience }\end{array}$ & $\begin{array}{l}\text { Quality of } \\
\text { Service }\end{array}$ & Network Performance \\
\hline $\begin{array}{l}\text { User behaviour } \\
\text { attribute }\end{array}$ & $\begin{array}{l}\text { Service } \\
\text { attribute }\end{array}$ & $\begin{array}{l}\text { Connection/Flow element } \\
\text { attribute }\end{array}$ \\
\hline $\begin{array}{l}\text { Focus on user } \\
\text { expected effects }\end{array}$ & $\begin{array}{l}\text { Focus on user } \\
\text { observable } \\
\text { effects }\end{array}$ & $\begin{array}{l}\text { Focus on planning, } \\
\text { development (design) } \\
\text { operations and } \\
\text { maintenance }\end{array}$ \\
\hline User subject & $\begin{array}{l}\text { Between (at) } \\
\text { service Access } \\
\text { points }\end{array}$ & $\begin{array}{l}\text { End to End or network } \\
\text { elements capabilities }\end{array}$ \\
\hline
\end{tabular}

\section{A. Delivery ratio and cellular overhead}

The delivery ratio and the equivalent routing visual projection for MSGPR, AODV, and DSR are exposed in figures 6 and 7. The routing overhead for MSGPR contains the routing requirements, routing reply, and point updates obtainable throughout the cellular radio channel. It is exposed that MSGPR has a much improved delivery ratio than AODV and DSR. The routing overhead in MSGPR is too much lesser. When the amount of active links enhance, the delivery ratio meant for MSGPR reduces, as is the case with AODV and DSR. This is appropriate to the improved conflict in the MAC coating. The overhead for MSGPR enhances slightly when the number of active links enhances because of the rising number of routing requirements and replies. 


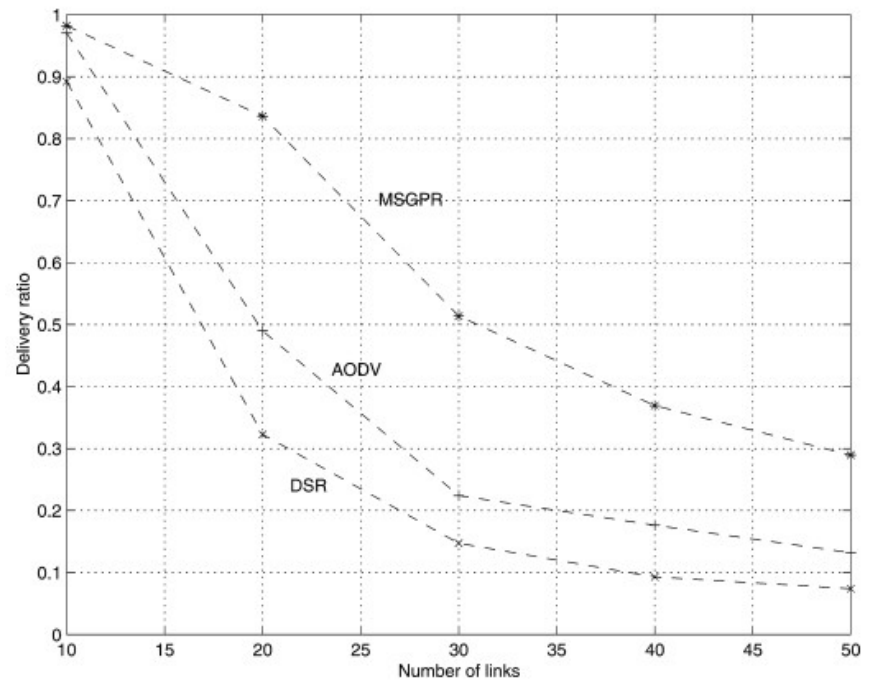

Fig. 7. Delivery ratio comparison among MSGPR, AODV, and DSR

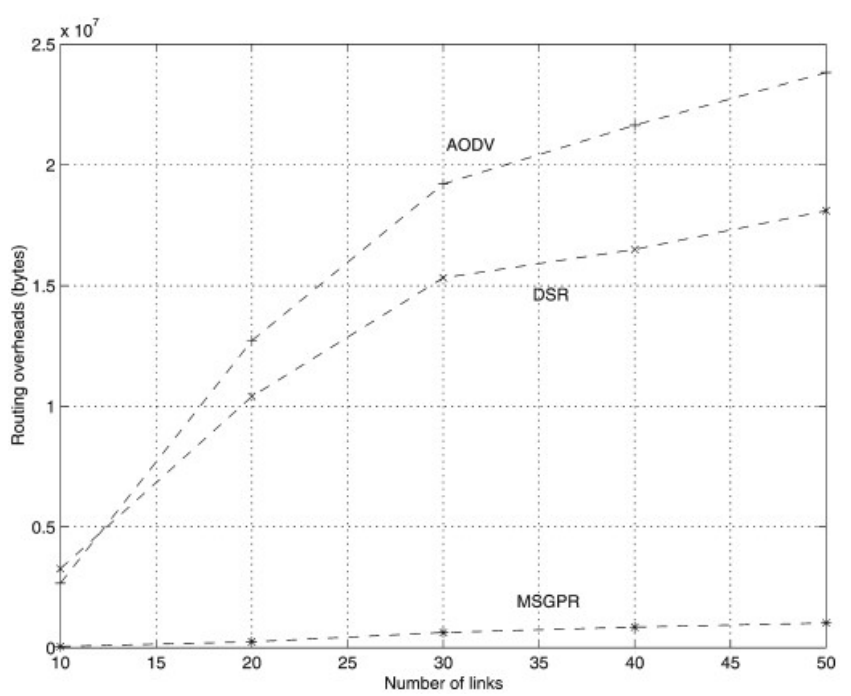

Fig. 8. Routing overhead comparison among MSGPR, AODV, and DSR

The routing visual projection within MSGPR is furthermore a cost in the cellular network. The expand by means of MSGPR over the AODV and DSR is exposed in figure 8 . The expand in the ad hoc networks (the number of supplementary bytes distributed effectively than would be in AODV and DSR) is just about 10 times as great as the cellular overhead at the middling network load and high network load. Intended for networks with short load, the expand is level bigger. Intended for commercial wireless services, it is significance by means of CAMA if a byte in a cellular network is down with than 10 times the assessment of a byte in an ad hoc network.

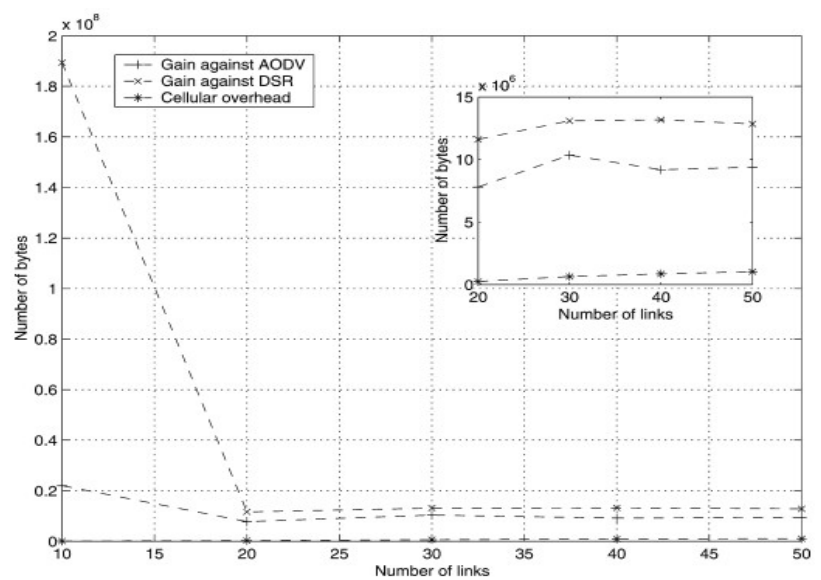

Fig. 9. Gains when using CAMA

\section{B. Packet delivery ratio}

We describe the packet deliverance ratio as the ratio among the number of packages established by the intention and the number of packages produced by the relevance layer sources of the conventional calls. Packet delivery ratio is significant as it illustrate the loss rate that will be observed by the transport protocols, which in revolve distress the maximum throughput that the network can sustain.

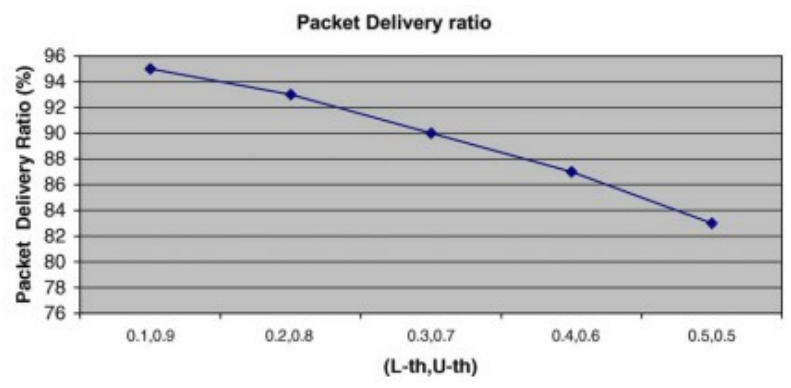

Fig. 10. Packet delivery ratio versus the link state update thresholds

The figure displays the integer of packets delivered effectively each second through the simulation phase. The probable successful deliverance rate of the sent package is five packets/ s. As of the throughput curvature, we can observe that network was capable to meet the potential at generally. From the figure, we capable also recognize or expect the packet loss in the network. Permanence of VGAP structural design (the virtual grid) permit it to accomplish elevated all through. Fig. 9 displays the packet delivery proportion by means of M-OSPF in VGAP and employing the link state announcement model that uses two threshold point. The thresholds (Lth, Uth) are distorted as of $(0.1,0.9)$ to $(0.5,0.5)$, correspondingly. VGAP is capable to attain high packet delivery ratio and continue good enough levels when the link updates enhance (enhance in control traffic). 


\section{End to End Delay}

The end-to-end packet delay is also studied. When a link is undependable, the node fails to promote packets, basis packet plunges or longer delays. On low traffic load, nodes infrequently experience obstruction but frequently knowledge broken links.

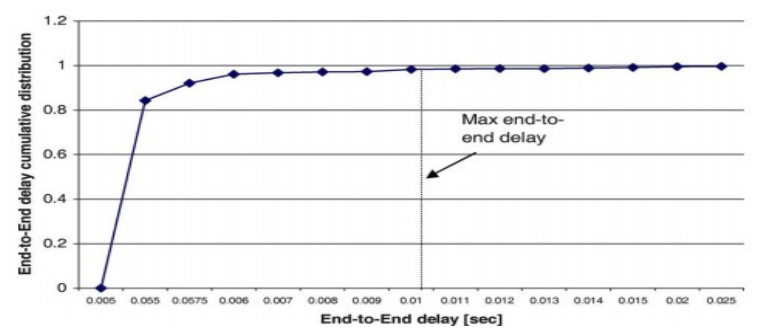

Fig. 11. Cumulative distribution of end-to-end packet delay

Consequently, packets that require to be re-routed will be queued and as a result encounter longer delays. The packet one after the other delay was situate to $10 \mathrm{~ms}$. VGAP was capable to convince the delay necessities for mainly of these packets as shown in Fig. 10. The outline shows the increasing prospect allocation of the continuous delay of the engender packets in the network. We observe that the majority packets were delivered effectively within their required delay bounds.

\section{DISCUSSION AND CONCLUSION}

The convergence of more than two wireless access networks, though more complex, has a better chance of guaranteeing the QoS of users' services and offers better optimization to overall network resource utilization. A flexible (not rigid) management mechanism, as evidenced in this article, plays a critical role in the success of such a complex network. The proposed protocol makes use of the communication of the underneath three layers of MANETs to develop the network concert and sustain of quality of service requirements. The protocol superimpose a usual rectilinear virtual topology on the substantial topology using a effortless zoning process that takes into deliberation the transmission range differentiation in a heterogeneous MANETs. Our simulation outcomes illustrate enhanced concert in terms of bandwidth and uninterrupted delay guarantees and further network toughness to link failure and topology differences. The protocol as well utilizes a power manage algorithm at the physical layer to afford communication among nodes and sustain network connectivity in this heterogeneous setting.

\section{FUTURE WORKS}

In future work we plan to extend the recovery model by using a hierarchical architecture with dedicated proxies in the assistant recovery network. Furthermore, we will investigate possible security implications that are introduced in the network due to the heterogeneity of two different networks. Finally we plan to implement the proposed protocol using socket programming in order to study its efficiency in a real environment.

\section{REFERENCE}

[1] Dinh, H. T., Lee, C., Niyato, D., \& Wang, P. (2013). A survey of mobile cloud computing: architecture, applications, and approaches. Wireless communications and mobile computing, 13(18), 1587-1611.
[2] Narayanan, V. A., Rajeswari, A., \& Sureshkumar, V. (2014). AN INTELLIGENT VERTICAL HANDOVER DECISION ALGORITHM FOR WIRELESS HETEROGENEOUS NETWORKS. American Journal of Applied Sciences, 11(5), 732.

[3] Wang, X., Vasilakos, A. V., Chen, M., Liu, Y., \& Kwon, T. T. (2012). A survey of green mobile networks: Opportunities and challenges. Mobile Networks and Applications, 17(1), 4-20.

[4] Yigitel, M. A., Incel, O. D., \& Ersoy, C. (2011). QoS-aware MAC protocols for wireless sensor networks: A survey. Computer Networks, 55(8), 1982-2004.

[5] Oh, H., Lee, J., \& Choi, J. K. (2013, October). Energy-efficient dynamic load distribution for heterogeneous access networks. In ICT Convergence (ICTC), 2013 International Conference on (pp. 18-23). IEEE.

[6] Yan, X., Ahmet Şekercioğlu, Y., \& Narayanan, S. (2010). A survey of vertical handover decision algorithms in Fourth Generation heterogeneous wireless networks. Computer Networks, 54(11), 1848-1863.

[7] Luo, Y., Tran, P. N., An, C., Eymann, J., Kreft, L., \& Timm-Giel, A. (2013, June). A Novel Handover Prediction Scheme in Content Centric Networking Using Nonlinear Autoregressive Exogenous Model. In Vehicular Technology Conference (VTC Spring), 2013 IEEE 77th (pp. 1-5). IEEE.

[8] Kantubukta, V., Maheshwari, S., Mahapatra, S., \& Kumar, C. S. (2013). Energy and quality of service aware FUZZY-technique for order preference by similarity to ideal solution based vertical handover decision algorithm for heterogeneous wireless networks. IET networks, 2(3), 103-114.

[9] He, D., Chi, C., Chan, S., Chen, C., Bu, J., \& Yin, M. (2011). A simple and robust vertical handoff algorithm for heterogeneous wireless mobile networks. Wireless Personal Communications, 59(2), 361-373.

[10] Mahoto, N. A., Shaikh, F. K., \& Chowdhry, B. S. (2014). Innovative Architecture to Enhance Quality of Service for Laboratory Management Information Systems. Laboratory Management Information Systems: Current Requirements and Future Perspectives: Current Requirements and Future Perspectives, 237.

[11] Klein, A., Lottermann, C., Mannweiler, C., Schneider, J., \& Schotten, H. D. (2011, June). A novel approach for combined joint call admission control and dynamic bandwidth adaptation in heterogeneous wireless networks. In Next Generation Internet (NGI), 2011 7th EURO-NGI Conference on (pp. 1-8). IEEE.

[12] Ren, S., Liu, Y., Zhou, X., Tang, H., \& Ci, S. (2013, July). A utility-based terminal selection mechanism for terminal cooperation in heterogeneous wireless networks. In Computers and Communications (ISCC), 2013 IEEE Symposium on (pp. 000989-000994). IEEE.

[13] Macone, D., Oddi, G., Palo, A., \& Suraci, V. (2013). A dynamic load balancing algorithm for quality of service and mobility management in next generation home networks. Telecommunication Systems, 53(3), 265-283.

[14] Bi, T., Trestian, R., \& Muntean, G. M. (2013). RLoad: Reputation-based load-balancing network selection strategy for heterogeneous wireless environments. In ICNP (pp. 1-3).

[15] Sadhukhan, P., Das, P. K., \& Mukherjee, N. (2013). A novel layer 3 based movement detection algorithm for improving the performance of mobile IP. Wireless networks, 19(4), 431-442.

[16] Kang, J. M., Strassner, J., Seo, S. S., \& Hong, J. W. K. (2011). Autonomic personalized handover decisions for mobile services in heterogeneous wireless networks. Computer Networks, 55(7), 1520-1532.

[17] Alkhawlani, M. M., \& Mohsen, A. (2012). Hybrid approach for radio network selection in heterogeneous wireless networks. Academic Journal, International Journal of Advanced Science and Technology, 44, 33-48.

[18] Ren, S., Liu, Y., Zhou, X., Tang, H., Ci, S., \& Wang, M. (2013, December). A novel peer selection mechanism in heterogeneous wireless peer-to-peer networks. In Networks (ICON), 2013 19th IEEE International Conference on(pp. 1-7). IEEE. 
[19] Giacomini, D., \& Agarwal, A. (2013). Optimizing end user QoS in heterogeneous network environments using reputation and prediction. EURASIP Journal on Wireless Communications and Networking, 2013(1), 1-13.

[20] Narayanan, V. A., Rajeswari, A., \& Sureshkumar, V. (2015). Service-Adaptive Fuzzy Multi Criteria Based Intelligent Vertical Handover Decision Algorithm for Heterogeneous Wireless Networks. In Artificial Intelligence and Evolutionary Algorithms in Engineering Systems (pp. 297-304). Springer India.

[21] Ernst, J. B., Kremer, S. C., \& Rodrigues, J. J. (2014). A Survey of QoS/QoE mechanism in heterogeneous wireless networks. Physical Communication.

[22] Shuminoski, T., \& Janevski, T. (2011). Novel adaptive QoS provisioning in heterogeneous wireless environment. International Journal of Communication Networks and Information Security (IJCNIS), 3(1).

[23] Bhuvaneswari, A., \& Raj, E. G. D. P. (2012). An overview of vertical handoff decision making algorithms. International Journal of Computer Network and Information Security (IJCNIS), 4(9), 55.

[24] Yigitel, M. A., Incel, O. D., \& Ersoy, C. (2014). QoS vs. Energy: A Traffic-Aware Topology Management Scheme for Green Heterogeneous Networks. Computer Networks.

[25] Jin, S., Xuanli, W., \& Xuejun, S. (2011, August). Load balancing algorithm with multi-service in heterogeneous wireless networks. In Communications and Networking in China (CHINACOM), 2011 6th International ICST Conference on(pp. 703-707). IEEE.

[26] Zhang, Y., Yu, R., Xie, S., Yao, W., Xiao, Y., \& Guizani, M. (2011). Home M2M networks: architectures, standards, and QoS improvement. Communications Magazine, IEEE, 49(4), 44-52.

[27] Haldar, K. L., Ghosh, C., \& Agrawal, D. P. (2013). Dynamic spectrum access and network selection in heterogeneous cognitive wireless networks. Pervasive and Mobile Computing, 9(4), 484-497.

[28] Lai, C. C., Liu, C. M., \& Su, Y. C. (2013, March). A novel mechanism to construct a compatible overlay on heterogeneous mobile peers. In
Pervasive Computing and Communications Workshops (PERCOM Workshops), 2013 IEEE International Conference on (pp. 78-83). IEEE.

[29] Yang, P., Sun, Y., Liu, C., Li, W., \& Wen, X. (2013, June). A novel fuzzy logic based vertical handoff decision algorithm for heterogeneous wireless networks. In Wireless Personal Multimedia Communications (WPMC), 2013 16th International Symposium on (pp. 1-5). IEEE.

[30] Bi, T., Trestian, R., \& Muntean, G. M. (2013, June). Reputation-based network selection solution for improved video delivery quality in heterogeneous wireless network environments. In Broadband Multimedia Systems and Broadcasting (BMSB), 2013 IEEE International Symposium on (pp. 1-8). IEEE.

[31] Shuminoski, T., \& Janevski, T. (2014). Radio Network Aggregation for 5G Mobile Terminals in Heterogeneous Wireless and Mobile Networks. Wireless Personal Communications, 1-19.

[32] Piamrat, K., Ksentini, A., Bonnin, J. M., \& Viho, C. (2011). Radio resource management in emerging heterogeneous wireless networks. Computer Communications, 34(9), 1066-1076.

[33] Ma, X., Liu, J., \& Jiang, H. (2011). On the design of algorithms for mobile multimedia systems: a survey. International Journal of Communication Systems, 24(10), 1330-1339.

[34] Márquez-Barja, J., Calafate, C. T., Cano, J. C., \& Manzoni, P. (2012, April). MACHU: A novel vertical handover algorithm for vehicular environments. InWireless Telecommunications Symposium (WTS), 2012 (pp. 1-8). IEEE.

[35] Nguyen-Vuong, Q. T., \& Agoulmine, N. (2011). Efficient Load Balancing Algorithm over Heterogeneous Wireless Packet Networks. REV Journal on Electronics and Communications, 1(1).

[36] Ma, B., Liao, X., \& Xie, X. (2012, October). Risk-aware vertical handoff algorithm for security access support in heterogeneous wireless networks. In Biomedical Engineering and Informatics (BMEI), 2012 5th International Conference on IEEE, (pp. 1515-1519). 